\title{
WHEAT LODGING ASSESSMENT USING MULTISPECTRAL UAV DATA
}

\author{
S. Chauhan ${ }^{1, *}$, R. Darvishzadeh ${ }^{1}$, Y.Lu ${ }^{1}$, D. Stroppiana ${ }^{2}$, M. Boschetti ${ }^{2}$, M. Pepe ${ }^{2}$, A. Nelson ${ }^{1}$ \\ ${ }^{1}$ Faculty of Geo-information Science and Earth Observation (ITC), University of Twente, Enschede 7500AE, The Netherlands - \\ (s.chauhan, a.nelson, r.darvish)@utwente.nl, y.lu-3@student.utwente.nl \\ ${ }^{2}$ CNR-IREA, Institute for Electromagnetic Sensing of the Environment, National Research Council, 20133 Milano, Italy - \\ (stroppiana.d, boschetti.m, pepe.m)@irea.cnr.it
}

Commission VI, WG VI/4

KEYWORDS: Lodging detection, Wheat, UAV, Red-edge, NIR, Reflectance

\begin{abstract}
:
Lodging is a major yield-reducing factors in wheat, causing reductions up to $80 \%$. Timely detection of lodging can reduce its impacts and support proper decisions regarding expected yield, crop price or its insurance. Since the incidence of lodging is heterogeneous within a field, very high-resolution remote sensing data can be viable for accurate and prompt spatio-temporal assessment of lodging severity. As such unmanned aerial vehicles (UAVs) provide a versatile and cost-effective solution to monitor crops on a small scale with sub-centimetre spatial resolution. In this study, we analysed the spectral variability between different grades of lodging severity (non-lodged (NL), moderate (ML), severe (SL) and very severe (VSL)) and classified them using high-resolution UAV data. Multispectral orthomosaic UAV images with $5 \mathrm{~cm}$ resolution and nine bands (covering the VIS-NIR spectrum with Sentinel-2 filters) were acquired in May 2018 for two wheat fields in Bonifiche Ferraresi farm, Jolanda di Savoia, Italy. Concurrent to the time of image acquisition, a field campaign was carried out in which crop characteristics and lodging related parameters were collected. The results showed that reflectance magnitude increased with lodging severity and demonstrated that the red-edge and NIR bands can be used to clearly discriminate between NL and lodged (all grades) wheat and to some extent between different lodging classes (ML, SL and VSL). The nearest neighbourhood classification performed using an object-based segmentation yielded optimal results with an overall accuracy of $90 \%$, thus demonstrating the use of multispectral UAV data as a promising tool for wheat lodging assessment.
\end{abstract}

\section{INTRODUCTION}

The lodging of cereal crops, which is caused due to genetic, crop management and environmental factors can have a detrimental impact on crop yield and grain quality (Berry et al., 2004). Especially in wheat, lodging is known to reduce crop yield by 60 80\% (Berry and Spink 2012; Setter, Laureles, and Mazaredo, 1997). Therefore, proper assessment of lodging can facilitate accurate yield estimation, help plan harvest operations, and aid in agricultural disaster relief compensation (Yang et al., 2017).

The traditional methods of assessing lodging rely on manual in situ measurements and visual inspection of lodged areas, which can be time-consuming, labour intensive and point-based. Remote sensing (RS) technology is a feasible and reliable alternative for obtaining timely information on crop lodging over vast areas (Atzberger, 2013). In the context of crop monitoring, unmanned aerial vehicles (UAVs) are increasingly being adopted as RS platforms (Colomina and Molina, 2014; Bendig et al., 2015). Compared to proximal systems, UAV platforms can survey areas more quickly without disturbing the vegetation (Burkart et al., 2015). In addition, their ability to provide data in high temporal, spatial and spectral resolutions along with their flexibility of operation, compared to aerial and satellite platforms, make them a promising tool for crop monitoring (Aasen et al., 2015).

Our ability to assess crop lodging with RS is directly affected by high within-field spatial variability of lodging and the need for fine spatial and spectral resolution data at low cost. It is only in the past decade that UAV data has been utilized to detect and assess lodging damage in crops and has shown great potential
(Chapman et al., 2014; Zhang, Walters, and Kovacs, 2014). While some studies have focused on the visual interpretation of lodging from orthomosaic images (Du and Noguchi, 2017; Zhang, Walters, and Kovacs,, 2014), a few others have dealt with quantitative lodging assessment (Chapman et al., 2014; Chu et al., 2017). Haiying, Guijun, and Hongchun (2014) reported that lodged wheat area could be extracted with an accuracy of more than $80 \%$ based on multi-spectral ( 3 bands) and textural features derived from UAV data. Yang et al. (2017), on the other hand, showed that adding texture information to the RGB image did not improve the lodging interpretation accuracy in rice. Recently, Liu et al. (2018) showed that the combination of visible, texture and thermal infrared images could be used to detect lodging in rice and estimate percentage of area affected with an accuracy of more than $90 \%$.

Some studies have demonstrated the feasibility of incorporating point cloud digital elevation or digital surface model (DEM or DSM) information for lodging assessment. For instance, Chapman et al. (2014) quantified the proportion of lodging in a wheat field by determining the variance of DEM-derived pixel heights. In another study, Chu et al. (2017) investigated the potential for lodging detection in maize using crop height estimated from UAV data together with structure-from-motion (SfM) photogrammetry. However, to date the number of RSbased studies characterizing crop lodging using UAV data is limited (Chauhan et al., 2019). Despite the interest, to the best of our knowledge, the sensitivity of multiple spectral bands (9) covering the entire $390-950 \mathrm{~nm}$ region of the spectrum to crop lodging has not been studied from UAV data. 


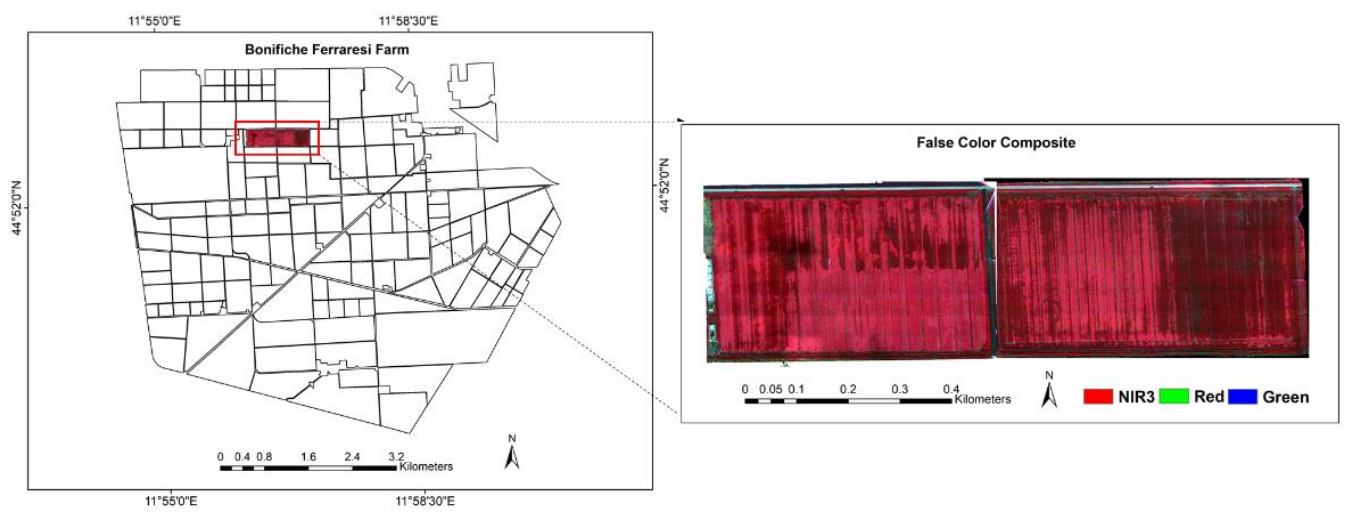

Figure 1. The selected wheat fields (red box) in Bonifiche Ferraresi farm, Jolanda di Savoia, Ferrara, Italy. The location map of the farm is shown on the left and the false colour composite (R: NIR3, G: Red, B: Green) of the UAV data is on the right

The main objective of this study was to analyse the spectral variability of the features derived from UAV data and their ability to discriminate between different grades of lodging severity: healthy/non-lodged (NL), moderate (ML), severe (SL) and very severe (VSL). Based on the analysis, we also tested an object based classification method to generate a thematic map of different grades of lodging severity.

\section{MATERIALS AND METHODS}

\subsection{Study area}

The study was conducted in the Bonifiche Ferraresi farm located in Jolanda di Savoia $\left(44^{\circ} 52^{\prime} 36.21^{\prime \prime} \mathrm{N}, 1^{\circ} 56^{\prime} 37.5^{\prime}\right.$ 'E), Ferrara, Italy (Fig. 1). The growing conditions (sowing date, crop variety, etc.) of the two selected fields were similar. Durum wheat (variety Marco Aurelio) was sown in both fields on 26 Oct 2017. The area covered by each field is 18.6 ha. The soil texture is mostly silty and clayey.

\subsection{Field data}

At the time of data acquisition, the crop was at the milking stage. The field data consists of observations about crop condition collected over multiple transects with a total of 51 plots of $2 \times 2 \mathrm{~m}$ geolocated by GPS measurements. We categorically defined four grades of lodging severity based on our visual assessment and crop angle/height measurements: healthy wheat with a crop angle $<5^{\circ}(\mathrm{NL})$, wheat with a crop angle between $5-30^{\circ}(\mathrm{ML})$, wheat with a crop angle between $31-60^{\circ}$ (SL) and wheat with a crop angle $>60^{\circ}$ (VSL). Of the 51 plots, 20 were NL while the remaining were categorized into different grades of lodging severity (ML, SL, and VSL). The field measurements were taken at the same time as UAV data acquisition.

\subsection{Platform and sensor}

The UAV flight was carried out by the SAL Engineering company. The UAV system was a dij s900 hexacopter with a global navigation satellite system (GNSS) and a MAIA S2 multispectral camera with nine monochrome sensors capable of the simultaneous acquisition of images at several wavelengths in visible (VIS) to near-infrared (NIR) region (390-950nm). Each sensor had a band-pass filter installed in the MAIA camera, with the same central wavelength and bandwidth as Sentinel-2. The extremely fast exposure time of the nine global shutter CMOS sensors (up to $1 / 5000 \mathrm{sec}$ ), low travel speed $(5 \mathrm{~m} / \mathrm{sec})$ and $100 \mathrm{~m}$ altitude from the ground guaranteed image acquisition without blurring effect.

The images were acquired with the ground sampling distance (GSD) of $5 \mathrm{~cm}$ and field of view of about $60 \times 45 \mathrm{~m}$. The images were acquired on a sunny day without strong wind between $10 \mathrm{am}$ and $4 \mathrm{pm}$.

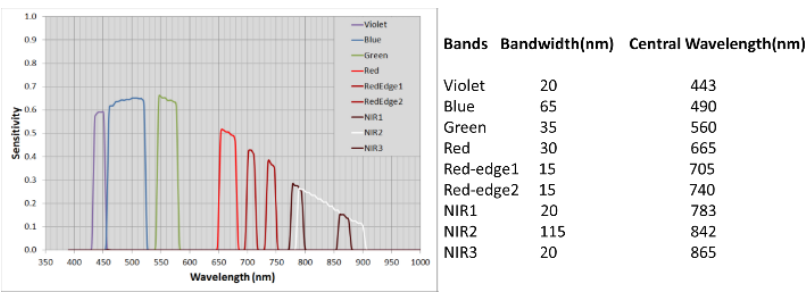

Figure 2. The sensitivity of optical bands of MAIA S2 cameras (Nocerino et al., 2017)

\section{METHODS}

\subsection{Data pre-processing}

The images were first geometrically corrected using the calibration certificate that is provided with each optic. The certificate contained the calibration interior orientation parameters that enabled the removal of geometric distortions. After that, the images were radiometrically corrected using an ILS (Irradiance Light Sensor). The images were acquired by setting the camera at 8 bit with the DN values (after correction) ranging between $0-255$. To obtain the reflectance (\%), the DN value was divided by 255 and then multiplied by 100 . The undistorted multilayer images were processed in Photoscan (Agisoft) software and the orthomosaic with nine bands was generated. The spatial resolution of the orthomosaic was $5 \mathrm{~cm}$.

\subsection{Extraction of spectral values and statistical analyses}

The average spectral values from the nine bands were extracted from the 51 plots. In order to account for edge effects, the plot size was reduced by $10 \mathrm{~cm}$ on each side. A 39x39 window was used and a total of 1521 pixels were averaged from each plot. The reflectance spectra of the sampled plots in nine bands (390$950 \mathrm{~nm})$ were first analysed for different lodging grades. A transect-based analysis was also performed by choosing a few 
samples of pixels to evaluate the radiometric variation of spectral reflectance for different grades (NL, ML, SL, VSL).

The Kruskal Wallis test was used to examine the statistical differences of sample means among the classes. This is a nonparametric test suitable for unequal sample sizes with more than two groups/classes.

\subsection{Classification of the target}

An object-based segmentation of the UAV multispectral orthomosaic was carried out in eCognition software using a multi-resolution segmentation (MRS) algorithm (Blaschke, 2010). It is a useful optimization approach for high-resolution images. Different scale (governs object size), shape (describes the similarity between object borders and a perfect square) and compactness (describes closeness of pixels) parameter values were tested to determine the optimal values. The image layer weights were set based on the test results of the spectral variability analysis.

We used supervised classification based on the nearest neighbour (NN) algorithm, whereby membership value between $0-1$ were selected based on the object's feature space distance to its nearest neighbour. Firstly, the field data were randomly divided into 32 plots for training and 19 for testing. A training and testing area (TTA) mask was created in eCognition and the classifier was trained using the training mask. The mean spectral reflectance from all bands for each identified object was used to classify the image. In addition, the normalized difference vegetation index (NDVI) was used as an independent masking criteria to separate soil patches from vegetation (NDVI <0.4). This part of the field corresponds to drainage or patches of visible soil as a consequence of lodging. To aid the selection of training samples, the sample editor window was used to provide a visual comparison of the separability between different classes according to mean spectral values. After classification, the image objects belonging to the same class were merged and exported.

The results of the classification were evaluated using two methods: classification stability (examines the ambiguity of the identified classes) and an error matrix. The error matrix was calculated using an independent test data (19 plots) and evaluated in terms of the overall accuracy, producer's (PA) and user's accuracy (UA) and kappa coefficient.

\section{RESULTS AND DISCUSSION}

\subsection{Spectra analysis for discrimination}

The spectral characteristics illustrated in Figure 3 provide a theoretical basis of the capability of remote sensing for discriminating lodging severity. Figure 3 a shows the box plot of the reflectance spectra for the four classes across different wavelengths. Lodging of wheat increased the magnitude of overall reflectance while following the same overall trend as a NL crop.

Considering the spectra of NL and lodged classes in general (Fig. $3 a)$, we can see that the relative change in spectral reflectance in the visible region is less than that in the red edge and NIR region (700-950nm). The overall reflectance in visible region is less due to increased chlorophyll absorption, except in the green band (central wavelength $560 \mathrm{~nm}$ ) where it's minimum. In the visible region $(390-700 \mathrm{~nm})$, the average reflectance of NL plants was $6 \%$ while that of VSL group was almost $9 \%$; while in red edge and NIR region, it increased to $13 \%$ and $23 \%$, respectively.

A red-edge band is the transition region (between red and NIR bands) of rapid change in canopy reflectance caused by strong chlorophyll absorption and scattering in NIR channel. It is highly sensitive in detecting physiological plant status (such as chlorophyll content), especially at high biomass and for detecting vegetation stress (Le Maire, Francois, and Dufrene, 2004). It is possible that a change in the crop physiological status during lodging makes the red-edge band sensitive to this phenomena. In the NIR region, however, the spectral gap between NL and the VSL group was the highest $(23 \%)$. This behaviour can be attributed to the change in plant structure (increase in crop angle) during lodging that increases reflectance as a consequence of more horizontal leaves condition. Thus, green $(560 \mathrm{~nm})$, RE1 (Red-edge1 $705 \mathrm{~nm}$ ), RE2 (Red-edge2 $740 \mathrm{~nm}$ ), NIR1 (Nearinfrared1 $783 \mathrm{~nm}$ ), NIR2 (Near-infrared2 $842 \mathrm{~nm}$ ), and NIR3 (Near-infrared3 $865 \mathrm{~nm}$ ) bands seem to better discriminate between NL and lodged crops than the other bands.

The variability in the mean spectral reflectance among the three grades of lodging is also shown in figure $3 \mathrm{a}$. The red-edge and NIR bands performed reasonably well in separating ML, SL and VSL groups. As can be seen in Figure 3a, on average, the reflectance for these three classes varied between $28 \%$ to $32 \%$ in the red-edge region $(705 \mathrm{~nm}, 740 \mathrm{~nm})$ and $51 \%$ to $58 \%$ in the NIR region $(783 \mathrm{~nm}, 842 \mathrm{~nm}, 865 \mathrm{~nm})$. On the right (Fig. 3b), we also show the transects of the pixels that were sampled from different
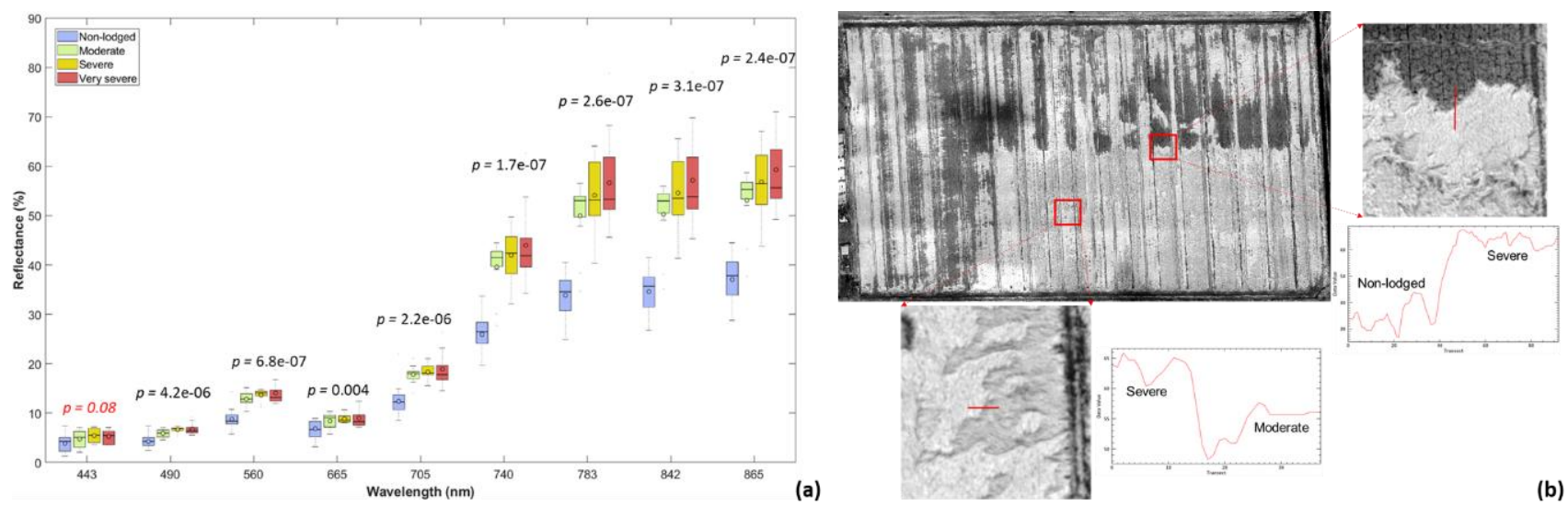

(b)

Figure 3. (a) Boxplot for field spectral reflectance at different wavelengths for different classes and (b) the transects of the pixels sampled from different classes in NIR1 $(783 \mathrm{~nm})$ band 
classes in NIR1 $(783 \mathrm{~nm})$ band. The above spectral analysis showed that the $770-950 \mathrm{~nm}$ range was statistically (at 0.01 significance level) the best wavelength interval for the lodging detection, followed by 700-750nm and 540-580nm. According to the Kruskal Wallis test, even though the other wavelength ranges (except 430-450nm) were also statistically significant, the relative change in the reflectance among different classes was very low.

\subsection{Lodging classification}

Given the different spectral patterns between the lodging severity groups, supervised classification is a logical choice for classifying the images. Using the field data as a reference, we chose the training samples (from the training mask) for different groups. The separability of two of the classes is shown in Figure 4.

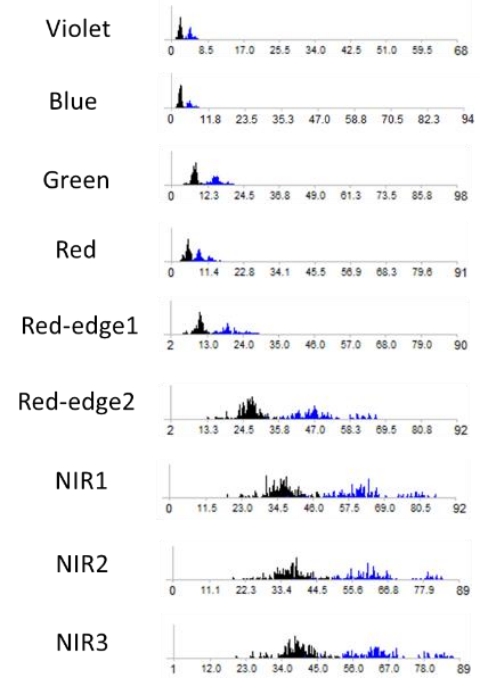

Figure 4. Separability between the training samples of nonlodged (black) and severe (blue) classes in different bands.

We then performed a nearest neighbourhood classification to identify lodged areas. A subset of these classified images is shown in Figure 5. Spectral analysis showed that green, red-edge and NIR bands were relatively more successful in discriminating between NL and lodged categories in general, and therefore more weights were assigned to these bands during the classification process. The soil target in the image has been denoted as 'Others' in Figure 5. The figure displays the results of clear discrimination of NL and lodged crop in general. According to the classified output, we found that around $58 \%$ of the total area was lodged (ML: 16\%, S: $28 \%$, VSL: 14\%), 32\% was non-lodged while the remaining belonged to 'Others' category.

\subsection{Accuracy assessment}

The accuracy assessment of the classification was performed in terms of classification stability (Table 1) and an error matrix (Table 2). The classification stability is calculated over all the image objects in the scene. The statistical operations are performed on the differences in degrees of membership of each object between the best and the second best class assignments.

The '\%Objects' column (Table 1) refers to the percentage of objects that were classified in a particular class. For instance, 31.36 and $13.93 \%$ objects were classified as NL and VSL class. 'Mean' corresponds to the mean difference between first and second best classification membership value for a particular class. "Min." and "Max." are the minimum and maximum values of the difference between first and second best membership values. The 'NL' and 'Others' class had reasonably good classification stability with a high mean of 0.124 and 0.301

\begin{tabular}{cccccc} 
Class & \%Objects & Mean & StdDev. & Min. & Max. \\
\hline NL & 31.36 & 0.1238 & 0.0896 & $3.87 \mathrm{e}-06$ & 0.452 \\
ML & 17.48 & 0.0407 & 0.0360 & $3.65 \mathrm{e}-06$ & 0.213 \\
SL & 24.66 & 0.0433 & 0.1024 & $9.53 \mathrm{e}-07$ & 1.000 \\
VSL & 13.93 & 0.0205 & 0.0187 & $1.19 \mathrm{e}-07$ & 0.123 \\
Others & 12.58 & 0.3014 & 0.2569 & $2.32 \mathrm{e}-06$ & 1.000
\end{tabular}
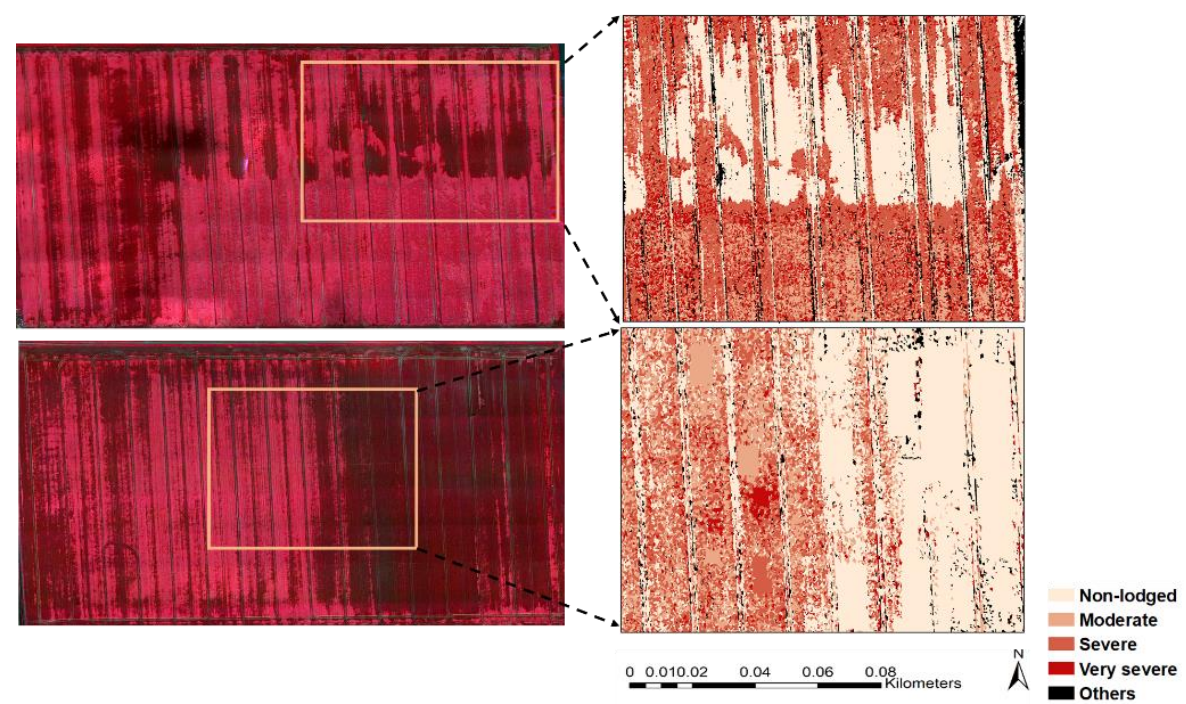

Figure 5. Detection of different grades of lodging severity from the UAV images 
respectively. For the other classes, the values are lower than 0.05 , which means that the second best assignment is nearly as good as the first. Thus, the classification stability among M, S, and VS groups is low as a consequence of the fuzzy transition between the lodged classes.

However, classification stability, by itself is not a sufficient determinant of a nearest neighbour classifier's performance. This is because the change in nearest neighbour slope function only affects the absolute membership values but not the sequence of membership to classes.

The accuracy of the classification was also assessed through an error matrix, based on the test data (ground truth). From Table 2, the overall accuracy of the classification was $90 \%$ while the kappa index of agreement (KIA) was 0.86 , which accounts the possibility of 'agreement occurring by chance.' Concerning the PA and UA for the individual classes, for "NL" and "Others," the accuracy is $>0.93$. On the other hand, PA and UA of the ML class are $74 \%$ and $80 \%$ respectively. According to the matrix, the classification results for NL, ML, SL, and 'Others' classes were reasonable.

A closer look at the matrix reveals that VSL class has a poor UA. There is significant mixing between SL and VSL classes. The most probable reason is that the spectral reflectance of VSL and SL classes is similar (a difference of only $2 \%$ ) and it is possible that the optical signal saturates when the crop angle is $>60^{\circ}$. Overall, unlike the NL class, there is a fuzzy transition between lodged classes and heterogeneity of lodging incidence can result in mixed pixels.

\begin{tabular}{lcccccc} 
Class & NL & ML & SL & VSL & $\begin{array}{l}\text { Other } \\
\text { s }\end{array}$ & Sum \\
\hline \multicolumn{7}{c}{ Confusion matrix } \\
NL & 37.24 & 0 & 0 & 0.13 & 0 & 37.37 \\
ML & 0.070 & 7.27 & 1.27 & 0.45 & 0 & 9.06 \\
SL & 0 & 0.65 & 14.58 & 0.35 & 0 & 15.59 \\
VSL & 0 & 1.95 & 3.52 & 4.06 & 0 & 9.54 \\
Others & 0.74 & 0 & 1.04 & 0 & 26.67 & 28.45 \\
Sum & 38.05 & 9.88 & 20.41 & 5.00 & 26.67 & 100
\end{tabular}

Accuracy (\%)

\begin{tabular}{|c|c|c|c|c|c|}
\hline $\begin{array}{l}\text { Produce } \\
\text { r }\end{array}$ & 98 & 74 & 71 & 81 & 100 \\
\hline User & 100 & 80 & 94 & 43 & 94 \\
\hline $\begin{array}{l}\text { KIA per } \\
\text { class }\end{array}$ & 96 & 71 & 0.66 & 79 & 100 \\
\hline
\end{tabular}

Totals

$\begin{array}{ll}\begin{array}{l}\text { Overall } \\ \text { accurac }\end{array} & 90 \% \\ \mathbf{y} & \\ \text { KIA } & 86\end{array}$

Table 2. Error matrix for the test areas

\section{CONCLUSIONS}

This study demonstrated the feasibility of using high-resolution UAV multispectral orthomosaic images to detect lodging in wheat, thereby allowing precision lodging management and understanding of on-going processes. The response behaviour of the reflectance spectra obtained from the UAV data was examined as a function of number of lodging severity groups. The discriminative features were explored through a comparison of the different grades of lodging severity (NL, ML, SL, and VSL). For the first time, high-resolution multispectral data from a UAV with nine spectral bands (the same as Sentinel-2) covering the 390-950 $\mathrm{nm}$ wavelength region has been utilized for lodging assessment. This enabled a comparison of spectral variability across nine bands. Overall, we found that there was an increase in the magnitude of reflectance spectra as the lodging became more severe. The increase was more pronounced in the green, red-edge and NIR regions of the spectrum, thereby showing the sensitivity of these bands to changes in the crop canopy structure. Furthermore, the overall classification accuracy was very high (90\%) where NL, ML, and SL classes were separated with reasonable accuracy while there was some mixing of VSL class with the other groups. To conclude, bands in the range of 700$950 \mathrm{~nm}$ can effectively detect lodging in wheat. These results underline how multispectral data can be a advancement with resepct to conventional RGB camera traditionally monted on the UAV platforms. Although we believe that these results are transferable to different crop varieties and growing conditions, further research is required to assess this. A comparative analysis using a digital surface model (DSM), textural features, and vegetation indices as inputs can be performed to examine if more separability can be attained, especially among the ML, SL, and VSL classes. Further efforts can also be directed towards a comparison of reflectance spectra from UAV data with that from a spectroradiometer and Sentinel-2.

\section{ACKNOWLEDGEMENTS}

The authors thank all those people who actively participated in the field campaign in 2018. We are grateful to Dr Donato Cillis of IBF-S technical team for his support and SAL Engineering team for UAV data acquisition and processing. The authors also thank the Bonifiche Ferraresi farm for hosting the experimentation and for supporting the field activities for the period $2017-2018$.

\section{REFERENCES}

Aasen, H., Burkart, A., Bolten, A., Bareth, G., 2015. Generating 3D Hyperspectral Information with Lightweight UAV Snapshot Cameras for Vegetation Monitoring: From Camera Calibration to Quality Assurance. ISPRS Journal of Photogrammetry and Remote Sensing, 108, 245-59.

Atzberger, C., 2013. Advances in remote sensing of agriculture: Context description, existing operational monitoring systems and major information needs. Remote sensing, 5(2), 949-981.

Bendig, J., Yu, K., Aasen, H., Bolten, A., Bennertz, S., Broscheit, J., Gnyp, M.L., Bareth, G., 2015. Combining UAV-Based Plant Height from Crop Surface Models, Visible, and near Infrared Vegetation Indices for Biomass Monitoring in Barley. International Journal of Applied Earth Observation and Geoinformation, 39, 79-87.

Berry, P. M., Sterling, M., Spink, J. H., Baker, C. J., SylvesterBradley, R., Mooney, S. J., Tams, A. R., Ennos, A. R., 2004. Understanding and Reducing Lodging in Cereals. Advances in Agronomy, 84, 217-71. 
Berry, P. M., Spink, J., 2012. Predicting Yield Losses Caused by Lodging in Wheat. Field Crops Research, 137, 19-26.

Blaschke, T., 2010. Object Based Image Analysis for Remote Sensing. ISPRS journal of photogrammetry and remote sensing, 65(1), 2-16.

Burkart, A., Aasen, H., Alonso, L., Menz, G., Bareth, G., Rascher, U., 2015. Angular Dependency of Hyperspectral Measurements over Wheat Characterized by a Novel UAV Based Goniometer. Remote sensing, 7(1), 725-46.

Chapman, S., Merz, T., Chan, A., Jackway, P., Hrabar, S., Dreccer, M., Holland, E., Zheng, B., Ling, T., Jimenez-Berni, J., 2014. Pheno-Copter: A Low-Altitude, Autonomous RemoteSensing Robotic Helicopter for High-Throughput Field-Based Phenotyping. Agronomy, 4(2), 279-301.

Chauhan, S., Darvishzadeh, R., Boschetti, M., Pepe, M., Nelson, A., 2019. Remote Sensing-Based Crop Lodging Assessment: Current Status and Perspectives. ISPRS journal of photogrammetry and remote sensing, 151, 124-40.

Chu, T., Starek, M., Brewer, M., Murray, S., Pruter, L., 2017. Assessing Lodging Severity over an Experimental Maize (Zea Mays L.) Field Using UAS Images. Remote Sensing, 9(9), 923.

Colomina, I., Molina, P., 2014. Unmanned Aerial Systems for Photogrammetry and Remote Sensing: A Review. ISPRS journal of photogrammetry and remote sensing, 92, 79-97.

Du, M., and Noguchi, N., 2017. Monitoring of Wheat Growth Status and Mapping of Wheat Yield's within-Field Spatial Variations Using Color Images Acquired from UAV-Camera System. Remote Sensing, 9(3), 289.

Haiying, L. I. U., Guijun, Y. A. N. G., Hongchun, Z. H. U., 2014. The Extraction of Wheat Lodging Area in UAV's Image Used Spectral and Texture Features. Applied Mechanics and Materials, 651-653, 2390-93.

Liu, T., Li, R., Zhong, X., Jiang, M., Jin, X., Zhou, P., Liu, S., Sun, C., Guo, W. 2018. Estimates of Rice Lodging Using Indices Derived from UAV Visible and Thermal Infrared Images. Agricultural and forest meteorology, 252, 144-54.

Le Maire, G, Francois, C., Dufrene, E. 2004. Towards Universal Broad Leaf Chlorophyll Indices Using PROSPECT Simulated Database and Hyperspectral Reflectance Measurements. Remote sensing of environment, 89(1), 1-28.

Nocerino, E., Dubbini, M., Menna, F., Remondino, F., Gattelli, M., Covi, D., 2017. Geometric Calibration and Radiometric Correction of the MAIA Multispectral Camera. International Archives of the Photogrammetry, Remote Sensing \& Spatial Information Sciences, 42.

Setter, T. L., Laureles, E. V., Mazaredo, A. M., 1997. Lodging Reduces Yield of Rice by Self-Shading and Reductions in Canopy Photosynthesis. Field Crops Research, 49(2-3), 95-106.

Yang, M. D., Huang, K. S., Kuo, Y. H., Tsai, H., Lin, L. M. 2017. Spatial and Spectral Hybrid Image Classification for Rice Lodging Assessment through UAV Imagery. Remote Sensing, 9(6), 583.

Zhang, C., Walters, D., Kovacs, J. M., 2014. Applications of Low Altitude Remote Sensing in Agriculture upon Farmers' Requests-
A Case Study in Northeastern Ontario, Canada. PLoS ONE, 9(11), 17-19. 